\title{
LA EXPERIENCIA SUBJETIVA EN LA INVESTIGACIÓN DE LA NEUROCIENCIA COGNITIVA. EL CASO DE LA NEUROFENOMENOLOGÍA
}

\author{
Sylvia Ordóñez \\ Instituto de Investigaciones Filosóficas Luis Villoro \\ Universidad Michoacana de San Nicolás de Hidalgo, México \\ sylviaordonezm@hotmail.com
}

Resumen

La relevancia de los informes subjetivos en la neurociencia cognitiva hace necesario conocer qué tipo de material experiencial se puede comunicar y observar subjetivamente. Una posibilidad de ese material está en las metodologías de primera y segunda persona, impulsadas por la neurofenomenología desde los años noventa. La historia del desarrollo de estas metodologías, en ocasiones negada o ignorada, puede esclarecer la utilidad de su incorporación a las investigaciones actuales de las ciencias cognitivas. Sus iniciadores partieron de la biología cognitiva, la fenomenología husserliana, la psicología cognitiva y las tradiciones meditativas orientales budista y védica, pero fueron abarcando un amplio rango de psicoterapias y de prácticas encaminadas al examen de "hacerse consciente" (becoming aware). En estos años de investigación se han encontrado patrones comunes entre todas esas técnicas analizadas, mediante una cooperación interdisciplinaria ejemplar.

Palabras clave: fenomenología, interdisciplina, metodologías, neurociencia cognitiva, subjetividad. 


\section{Subjective Experience in Neurocognitive Science The Case of Neurophenomenology}

Abstract

Due to the relevance of subjective reports in cognitive neuroscience, it is presently needed to acknowledge the kind of data that can be subjectively observed and reported. One stance of these data is found in first and second person methodologies, developed in Neurophenomenology since the nineties. The history of these developments, sometimes ignored or denied, may clarify their relevance to cognitive science. The roots of these methodologies are found in cognitive biology, husserlian phenomenology, cognitive psychology and Eastern Buddhist and Vedic meditation techniques, but later included a wide range of related fields such as psychotherapies and different approaches to methods for "becoming aware". Research in all those areas allowed finding common patterns among the different fields as a result of exemplary interdisciplinary cooperation.

Keywords: Cognitive Neurosciences, Interdiscipline, Methodologies, Phenomenology, Subjectivity. 
Nuestro objetivo es presentar un camino para la exploración de la experiencia subjetiva desarrollado dentro del programa de investigación de la Neurofenomenología (Nf), que vincula la experiencia subjetiva con datos neurofisiológicos. Esta historia queda situada dentro del panorama de desarrollos de la exploración de diversos aspectos de la subjetividad. Nos percatamos de que en la actualidad la exploración de la subjetividad es fundamental para las ciencias cognitivas. Creemos que esta visión histórica permitirá comprender mejor la importancia de la incorporación de diferentes aspectos de la subjetividad a la neurociencia actual.

Nuestra hipótesis es que el análisis de la historia del desarrollo de metodologías para el abordaje de la experiencia subjetiva desde el punto de vista de la fenomenología y otras disciplinas afines, puede ayudar a la comprensión de su génesis y de la realidad de su aplicación en diversos campos de la neurociencia. En particular pretendemos destacar y discutir los logros epistémicos en su evolución que pueden permitir que los datos encontrados por estas metodologías obtengan validez dentro de la neurociencia cognitiva actual. Queremos refutar la idea de que estas exploraciones de la subjetividad están poco desarrolladas, pues mostraremos su historia con su génesis y evolución, basadas en buena parte en tradiciones tan recientes como la fenomenología husserliana y tan antiguas como las milenarias tradiciones meditativas orientales.

Las metodologías actuales para la exploración de la experiencia subjetiva son diversas. Sólo mencionaremos como muestras la heterofenomenología (Dennett, 2001), el muestreo de momentos subjetivos (Hurlburt, 2006) y el método narrativo para la investigación de la conciencia (Díaz, 2007; 2013), desarrollados a partir de la inquietud de tener formas confiables de obtener informes en primera persona.

1 Agradezco el apoyo y la asistencia del Dr. Antonio Zirión Quijano, así como la colaboración del Dr. Eduardo González Di Pierro. 
Aquí presentaremos las propuestas que se han hecho dentro del programa de investigación de la Neurofenomenología para impulsar y desarrollar metodologías de primera y segunda persona en el estudio y descripción de las vivencias. ${ }^{2}$ Inicialmente, estas metodologías fueron un intento de "proveer las bases para una ciencia de la conciencia que incluyera la experiencia subjetiva de primera persona como un componente activo y explícito" (Varela \& Shear, 1999: p.2). La inclusión del aspecto de la segunda persona ${ }^{3}$ fue posterior, según se relatará en esta exposición histórica.

Nos han interesado estas propuestas porque creemos que han logrado avances tangibles en un campo de nuestro interés, que es el de la epilepsia, en investigaciones sobre la anticipación de crisis y de auras epilépticas (Petitmengin, 2007a; Le Van Quyen, 2010). Estas investigaciones toman en cuenta el dominio de las vivencias desde el punto de vista de la fenomenología (aspectos fenodinámicos), y aspectos neurofisiológicos complejos a gran escala (neurodinámicos), sin posiciones reduccionistas.

Reiteramos, como hipótesis, que el análisis de la historia del desarrollo de las metodologías para el abordaje de las vivencias desde el punto de vista de la fenomenología, puede ayudar a una mejor comprensión de su génesis y de la realidad de su aplicación y utilidad en diversos campos de la neurociencia cognitiva.

En una edición reciente de Principles of Neural Science (Kandel, 2012) encontramos que Cristopher Frith (2012) plantea una apertura a la aceptación en la neurociencia cognitiva contemporánea de los informes subjetivos. En vista de que este autor señala, en relación a la declinación del enfoque conductista, que los métodos para registrar los informes subjetivos "se rezagaron considerablemente en relación a los métodos para estudiar la conducta aparente" (Frith, 2012: p. 1383), nos alarma que ignore o haga a un lado otros abordajes de la experiencia subjetiva. Menciona, pero descalifica, los intentos de

2 Los textos ingleses traducen el alemán Erlebnis por "experiencia vivida", pero el español ha acuñado una expresión exacta de corte fenomenológico: "vivencia".

3 Como se verá posteriormente, consiste en la intervención de un mediador en la promoción, obtención y descripción de la experiencia en primera persona. 
introspección, y ni siquiera menciona la ya centenaria fenomenología de Husserl ni las milenarias tradiciones meditativas orientales.

Por lo tanto, para argumentar en contra de lo asentado por Frith, en una obra legitimadora de métodos para las neurociencias, nos interesa exponer la historia de metodologías susceptibles de ser aceptadas y validadas para protocolos neurocientíficos actuales.

Frith sí acepta la complementariedad de una contraparte subjetiva para los estudios de neuroimagen. Considera que son una forma de verificación y apunta que puede detectarse actividad neural en zonas localizadas del cerebro durante la actividad mental que no se acompaña de una conducta externa. Asimismo señala que el contenido de actividad mental como la imaginación o la ensoñación sólo puede conocerse por informes verbales.

Las historias que presentaremos pretenden aclarar y resaltar la enorme omisión o descuido de Frith, pues nos parece que la plena aceptación de metodologías para el abordaje de la experiencia subjetiva se puede apoyar en la historia - predominantemente de un desarrollo epistemológico de las metodologías impulsadas por la Neurofenomenología como programa de investigación-, en el intento de conjuntar la investigación de la experiencia subjetiva con datos neurofisiológicos.

II.

Los primeros esbozos del desarrollo de las metodologías para el abordaje de las vivencias los encontramos en el enfoque fenomenológico de Francisco Varela, en sus propuestas iniciales para la Neurofenomenología. Según Rudrauf (2003), Varela propuso explorar experimentalmente la actitud de "hacerse consciente" (becoming aware) de sí mismo.

En 1996, Varela publica el artículo "Neurophenomenology: A Methodological Remedy for the Hard Problem", en una revista que aceptaba la vinculación entre la fenomenología y los estudios 
científicos de la conciencia, el Journal of Consciousness Studies. ${ }^{4}$ Varela propone un programa de investigación con características novedosas para su época. Lo denomina "Neurofenomenología", término que había sido acuñado previamente por el antropólogo Charles Laughlin (1990). Varela enuncia su denominación del programa de investigación:

Neuro-fenomenología es el nombre que uso aquí para designar la investigación para casar la moderna ciencia cognitiva y un enfoque disciplinado de la experiencia humana, colocándome así en el linaje de la tradición continental de la fenomenología (Varela, 1996: p.330).

Se refiere asimismo a la empresa de la investigación del "problema difícil" de la conciencia y la "brecha explicativa" entre los procesos neurobiológicos y la experiencia subjetiva abordada por la fenomenología. ${ }^{5}$ Plantea la necesidad de una nueva comunidad de investigación con innovadoras herramientas pragmáticas para afrontar los retos de la ciencia de la conciencia. Enfatiza que no se requiere más teoría, sino la "exploración sistemática" de la "estructura de la experiencia humana", por ser "el único vínculo entre mente y conciencia que parece obvio y natural" (Varela, 1996: p. 330). ${ }^{6}$

Varela inicia su propuesta con una aclaración sobre su posición: no se considera adscrito "a ninguna escuela o sub-linaje sino que representa mi propia síntesis de la fenomenología a la luz de la moderna ciencia cognitiva y otras tradiciones que se enfocan en la experiencia humana" (Varela, 1996: p.335). Consideramos que él basa su

4 Este mismo artículo ha aparecido recientemente en diversas publicaciones europeas (Capuccio, 2006; Gladziewjeski, 2010) como si fuera contemporáneo. Aunque este artículo podría considerarse "clásico", es problemático que se presente con una actualidad que no tiene, pues ello opaca el conocimiento de sus desarrollos ulteriores.

5 El problema "difícil" (hard) de la conciencia se refiere a la imposibilidad de explicar los datos fenomenológicos a partir de los datos neurales.

6 Ha de entenderse que aquí "mente" se refiere a ciertos fenómenos vistos desde el lado de las ciencias cognitivas, y"conciencia" al campo de estudio de la fenomenología. (Aclaración sugerida en comunicación personal por Antonio Zirión). 
propia síntesis de la fenomenología en los dos puntos fundamentales siguientes.

El primer punto consiste en colocarse "a la luz de la moderna ciencia cognitiva”. Aquí creemos que recoge toda su tradición personal que se remonta a las investigaciones sobre la autopoiesis que realizó con Maturana desde los años sesenta (Maturana y Varela, 1987). Esta tradición prosigue con sus aportaciones al desarrollo de las ciencias cognitivas, principalmente con el concepto de enacción ${ }^{7}$ contrapuesto al paradigma cognitivista de representación, y que aparece ya claramente delineado en obras de los años ochenta (Varela, 1988). Como recapitulación, y como preámbulo a su artículo clásico de 1996, Varela elaboró una de sus obras más difundidas, (De cuerpo presente, 1991), que escribió con Evan Thompson, y donde desarrolla la mayor parte de los conceptos que van a dar lugar al lanzamiento de la propuesta de la neurofenomenología en 1996. Todo esto abarca una recapitulación de más de treinta años de investigación.

El segundo punto que consideramos fundamental en la introducción que hace Varela de su "síntesis personal de la fenomenología” es la mención de otras tradiciones de estudio o reflexión sobre la experiencia humana. Aquí creemos que alude a las tradiciones orientales, las cuales no aborda en el artículo de lanzamiento de la Neurofenomenología en 1996, pero que ya había desarrollado extensamente en The Embodied Mind de 1991. Ambos puntos son vitales para esclarecer los planteamientos del enfoque fenomenológico de Varela.

El artículo de 1996 ofrece un enfoque fenomenológico que llama la atención por la concisión con que Varela aborda aspectos fenomenológicos de enorme complejidad, planteando un método aparentemente sucinto que a primera vista puede parecer una sobresimplificación de los desarrollos husserlianos y del movimiento fenomenológico en general. Varela aclara que se trata de su propia

7 Concepto nuevo propuesto por Varela para la cognición, no como procesamiento de información y basado en la representación de un mundo pre-dado, sino como un "hacer emerger" un mundo. 
síntesis de la fenomenología, reconociendo el papel de Husserl como iniciador en el Occidente.

Varela concibe a la fenomenología como un "tipo especial de reflexión o una actitud sobre nuestra capacidad de ser o estar conscientes" (Varela, 1996: p. 335). Señala que el punto "arquimédico" de la fenomenología es "suspender nuestras pretensiones habituales para catalizar un examen fresco" (Varela, 1996: p. 336). Enfatiza que la base de la fenomenología está en esa actitud específica de reflexión llamada reducción fenomenológica. Propone analizar el cambio de la actitud natural a la actitud fenomenológica a través de cuatro aspectos o momentos entretejidos: 1) la actitud de reducción, 2) la intuición y las variaciones imaginarias, 3) la descripción con sus invariantes y 4) el entrenamiento para el cultivo disciplinado de la reducción fenomenológica.

La primera impresión sobre esta parte del artículo obliga a pensar en una audaz sobresimplificación. Por más que se argumente una “síntesis personal", llama la atención que Varela invite a un abordaje de las vivencias de una forma tan simple. Nuestra explicación podría ser que trató tanto de captar el interés de los lectores que desconocían la fenomenología así como de presentar un panorama más atractivo para los que tuvieran reservas sobre la fenomenología, en virtud de su muy notoria complejidad y la difícil lectura de sus obras básicas. Otra explicación podría ser que, estando ya en marcha el plan de desarrollo de las nuevas metodologías fenomenológicas, Varela no quiso adelantar información sobre una investigación todavía confusa e incompleta.

Al final de la exposición de "su" enfoque fenomenológico, Varela hace algunos señalamientos. Indica que está consciente de que existe poca literatura publicada sobre la reducción fenomenológica. ${ }^{8}$ Considera que eso es un "síntoma y una causa de la escasez de los trabajos recientes sobre los enfoques fenomenológicos de la mente" (Varela, 1996: p.341). Insiste en que comprende que el lector no

8 Esto es inexacto. Probablemente Varela se refiere a una escasez en el campo de la literatura de la ciencia objetivista y no al de investigación en fenomenología propiamente dicha. 
pueda tener una buena idea de la reducción fenomenológica, que considera "el centro del remedio metodológico" que ofrece en el título del artículo. Enfatiza que es llamativo que se haya prestado tan poca atención a la "capacidad de hacerse consciente (becoming aware) como una pragmática humana” (Varela, 1996: p.341). Habla de la necesidad del desarrollo de estrategias para la reflexión de "inspiración fenomenológica”, y menciona dos fuentes potenciales para ese logro: la práctica de la investigación cognitiva de Vermersch y la introspección y las tradiciones de "mindfulness" de las diversas escuelas meditativas orientales, ya exploradas por el mismo Varela junto con Thompson en The Embodied Mind. Varela señala:

Mi único comentario al enfrentar la pobreza relativa de la elaboración pragmática es que representa un llamado urgente a una investigación que llene la brecha. Mi propia contribución en relación a la práctica de la reducción y su entrenamiento se presentará en un próximo trabajo en colaboración (Varela, 1996: p.341).

Varela ya había conjuntado esfuerzos con investigadores de diversos campos para iniciar el desarrollo de metodologías para el abordaje fenomenológico de las vivencias y su vinculación con la investigación de la neurociencia cognitiva. Los resultados y nuevas rutas aparecieron durante los años siguientes al artículo de 1996. Los propósitos de desarrollo de metodologías para el abordaje de la experiencia subjetiva se cumplieron en varias publicaciones. Varela también logró crear nuevas comunidades de investigación, ya que participó en grupos ${ }^{10}$ que se ocuparon simultáneamente de las dos vertientes de la neurofenomenología, o sea, de los aspectos neurocientíficos y de los aspectos fenomenológicos, con la meta de establecer puentes significativos entre ambos. Asimismo esos dos dominios deberían ejercer limitaciones o constreñimientos mutuos, pero

9 En De cuerpo presente (traducido en 2005) aparece como"presencia plena, conciencia abierta".

10 En el Hospital de la Salpetriere en Paris fundó el grupo "Brain Dynamics Team in the Cognitive Neurosciences and Brain Imaging Laboratory", que sigue vigente. 
también iluminarse entre sí. Aquí debe apuntarse que Varela siempre situó esas dos vertientes dentro del contexto de la Teoría de Sistemas Dinámicos.

III.

La siguiente obra que trataremos en la historia de los desarrollos de las metodologías impulsadas por la neurofenomenología para el abordaje de las vivencias inició su gestación en 1994, cuando sus promotores se reunieron en el seminario "Psychologie et Phenoménologie” organizado por el psicólogo Pierre Vermersch. Ahí participaron también Varela, biólogo cognitivo, y Nathalie Depraz, filósofa especialista en Husserl, con ponencias que encontraron una "resonancia entre sus textos" en discusiones intensas, lo que los llevó a la decisión de publicar una obra conjunta. La "chispa unificante" fue el trabajo de Vermersch, como contexto para las discusiones que condujeron a un manuscrito tentativo, de final abierto, que fungió como la semilla del libro On Becoming Aware. A Pragmatics of Experiencing (OBA) (Depraz, 2003).

El libro de Depraz y compañía (OBA) apareció en 2003, después de la muerte de Varela en 2001. Sus autores, provenientes de distintas disciplinas pero todos en contacto con las técnicas meditativas orientales, describen la creación de la obra como "un viaje de descubrimiento [...] una voz colectiva [...] una ejecución a seis manos con responsabilidades equitativamente repartidas" (Depraz, 2003: p.6). La pretensión fue ir tras las fuentes y los medios para lograr un enfoque disciplinado práctico para investigar la experiencia humana.

No se pretendió plantear a priori una nueva teoría de la experiencia, sino "describir una actividad, una praxis concreta, el investigar la actividad consciente en la medida que se percibe a sí misma desenvolviéndose en un modo operativo e inmanente, a la vez habitual y pre-reflectivo"11 (Depraz, 2003: p.1). Se trató de comprender

11 El término "pre-reflective", usado en el libro, se traducirá con el neologismo "pre-reflectivo". 
“cómo examinamos lo que vivimos" y el hacerse consciente (becoming aware) de nuestra propia vida mental.

En esta historia de las metodologías de primera y segunda persona, impulsadas por la neurofenomenología, es importante puntualizar la trayectoria de los términos "pre-reflected" (pre-reflejado) y "pre-reflective" (pre-reflectivo), que son relativamente conflictivos, sobre todo en su traducción. En el principio de $O B A$ se señala que el tipo de "reflexividad" para explorar el nivel "no reflejado" de la vida requiere preguntarse sobre esa zona "pre-reflectiva y pre-dada de nuestra subjetividad", para hacerla consciente (Depraz, 2003: p.2). Más adelante se dice que el acto de "hacerse consciente" (becoming aware) puede recibir varios nombres y en inglés se habla de un "reflecting act" que equivale en el contexto fenomenológico a "reducción". A continuación aclaran:

El término "reflecting act" es un término técnico para nosotros. Lo distinguimos, así como el acto mental específico que designa, de "reflection", "reflexive" y "reflective act". Para evitar rigidez estilística también usamos como sinónimo de "reflecting act”, la frase en inglés "becoming aware" aunque la expresión francesa "la prise de conscience" tiene una connotación más claramente psicológica (Depraz, 2003: p.15). ${ }^{12}$

En $O B A$ se incluyeron todas las dimensiones ordinarias de la vida humana (percepción, movimiento, memoria, imaginación, habla, interacciones sociales cotidianas) y todos los eventos cognitivos que pudieran ser "definidos en forma precisa como tareas en experimentos de laboratorio” (Depraz, 2003: p.2). Aquí vemos claramente el propósito de vincular estas exploraciones con la investigación científica convencional.

12 Posteriormente en el libro, y tomando en cuenta la mezcla de elemento husserlianos, psicológico-introspectivos y de meditación, se habla de una "reflexión cognitiva (cognitive reflection) que parte de la conciencia pre-reflectiva (pre-reflective conscience) (pre-discursiva, pre-noética, ante-predicativa, tácita, pre-verbal, pre-lógica, o no-conceptual...)" (Depraz, 2003: p.42). 
Fue importante para esta obra abordar la experiencia en el sentido de lo vivido de primera mano. Se habla de un énfasis "no en el contexto de la acción sino en la naturaleza inmediata, encarnada, y personal de los contenidos de la acción" (Depraz et al., 2003: p.2). Se define a la experiencia como aquello a lo que un sujeto singular está sujeto en cualquier tiempo y lugar, a lo que tiene acceso "en primera persona”. La experiencia es precisa, concreta e individualizada y centrada en parámetros espacio-temporales particulares. Se trata de una vida que se vive interiormente y en relación con el mundo exterior (en términos fenomenológicos: inmanente $e$ intencionalmente). Se relaciona consigo misma y con los objetos, sean perceptivos, afectivos o aperceptivos (Depraz, 2003: p.3).

Los autores se propusieron hablar en términos de varias disciplinas, de "informes de primera mano", "acceso de primera persona”, "introspección”, "datos fenomenales", o "vivencias (vecus, Erlebnisse)". Incluyeron aspectos de lo inconsciente, lo preconsciente, lo pre-reflectivo, lo auto-presente (self-present), lo pre-noético, lo pre-predicativo. En esta variedad se buscó encontrar diferencias y articulaciones (Depraz, 2003: p.3).

De diferentes dominios como la neurociencia cognitiva, la fenomenología, la psicología cognitiva, diversos tipos de psicoterapias y prácticas meditativas, que privilegian "el examen de la conciencia”, se buscó lo que comparten y sus patrones comunes, así como la integración de sus diferentes discursos, pero se consideró que los resultados obtenidos tendrían valor sólo si investigaban y aportaban los medios de alcanzar la experiencia característica de esos campos, explorada a través de una práctica disciplinada (Depraz, 2003: p.3).

Hay una búsqueda por encontrar la manera como en la neurociencia cognitiva surge la necesidad de contar con los datos de primera persona, la necesidad de la reducción como una praxis concreta y encarnada en la fenomenología y, por otro lado, la manera como se manifiesta la necesidad de una introspección en la psicología cognitiva y la necesidad de saberes en una variedad de psicoterapias, incluyendo requerimientos semejantes en las tradiciones meditativas orientales. Sería imposible detallar las obras involucradas, por lo que ofrecemos sólo pinceladas ilustrativas del contenido de las mismas, 
pues por ahora nos interesa que se tenga una visión histórica de conjunto.

Presentamos sólo una visión esquemática de la dinámica de hacerse consciente, la cual comprende lo siguiente: 1) Movimiento de epojé como una suspensión inicial, ${ }^{13}$ que debe repetirse en cada paso. 2) Reconocimiento de la evidencia intuitiva como criterio de verdad interna a cada acto. 3) Expresión del contenido de cada acto. 4) Validación intersubjetiva de los hallazgos en la expresión del contenido de cada acto. 5) Hacerse consciente de la temporalidad multi-estratificada de cada acto (Depraz, 2003: p.6). ${ }^{14}$

Podemos ver en los pasos señalados una similitud con la versión de la "síntesis personal" de la fenomenología expuesta en el artículo de Varela de 1996. Es evidente la "inspiración” husserliana. Aunque la psicología experimental y las ciencias cognitivas se habían abierto para considerar contenidos mentales y reportes verbales, éstos no abarcaban el rango completo de la experiencia vivida personal. Se propuso entonces volver a la introspección tras revisiones y actualizaciones de la misma. Para esto se planteó el requerimiento de desarrollar metodologías renovadas y rigurosas, y "descripciones precisas de la experiencia subjetiva” (Depraz, 2003: p. 7).

La obra descriptiva de Husserl se consideró una fuente de inspiración y se pretendió darle un sitio en un programa de investigación psicológica. Por lo tanto se buscó una apertura a una "psicofenomenología". Los autores declaran que pretendieron que su obra permitiera no sólo un nuevo despertar de la psicología introspectiva. También buscaron “apropiarse de nuevo y renovar" el enfoque fenomenológico (Depraz, 2003: p. 7).

13 En el original plantean que este esquema es todavía "misterioso", pero que se tratará de ir desarrollando y explicando. Posteriormente se aclara en qué consiste: "suspender el prejuicio 'realista' de que lo que aparece para ti es realmente el estado del mundo. Esta es la única forma de cambiar el modo como se pone atención a tu propia vivencia; en otras palabras, debes romper con la 'actitud natural'"' (Depraz, 2003: p. 25).

14 Aunque estos aspectos son difíciles de esquematizar, hacerlo es necesario en el contexto de este trabajo para tener la panorámica histórica de los desarrollos de estas metodologías. En vista de la mezcla de corrientes y tradiciones no pueden hacerse definiciones estrictas de la terminología. Todo esto sigue en proceso en la actualidad. 
Los autores manifiestan que les preocuparon los malentendidos sobre el término "fenomenología" debido a las variantes con que se ha empleado en una diversidad de campos. En la filosofía se refieren a Husserl, Heidegger, Merleau-Ponty, Sartre, Levinas y Henry, y entre los psicólogos y sociólogos se mencionan las obras de Schütz y Garfinkel, con interés en describir y categorizar la experiencia concreta. Entre los científicos cognitivos el término "fenomenología" se ha usado como la posibilidad controvertida de "un acceso de primera persona a datos que sean científicamente creíbles, o sea, objetivos" (Depraz, 2003: p. 7).

En esta obra se consideró que aunque el término "fenomenología” se había usado en los textos tradicionales, y en relación con la experiencia concreta en primera persona, ellos pretendieron ampliar el sentido de la experiencia, pero "centrada en su singularidad concreta, y con referencia [...] a su praxis y a su descripción procedimental" (Depraz et al., 2003: p. 8). De este modo el término "fenomenología", que se invoca aquí, tiene un rango de significado relacionado con las ciencias humanas y cognitivas, pero vinculado con los recursos de "rigor y sentido" de la filosofía fenomenológica.

Los autores se adelantaron a considerar la objeción fundamental de invocar una posible deformación de la experiencia al ser examinada, tema que será retomado intensivamente en la historia del desarrollo de las metodologías de primera y segunda persona. Reconocieron una dimensión hermenéutica en el manejo de los datos fenomenológicos en la exploración de la experiencia, y la necesidad de que la obtención de datos estuviera sujeta a una validación intersubjetiva.

Al conjugarse las visiones de tres investigadores de distintas disciplinas se hizo indispensable un glosario de términos de las áreas cognitivas, fenomenológicas y de budismo tibetano, que complementaron las exploraciones y discusiones interdisciplinarias constitutivas del cuerpo de la obra en sus aspectos eminentemente pragmáticos. 
IV.

En On Becoming Aware: A Pragmatics on Experiencing se logró un entretejido de posturas y una vinculación de disciplinas que permitieron propuestas para la exploración sistemática de la experiencia vivida, así como de la forma de hacerse consciente de ella mediante un aprendizaje y un entrenamiento también sistemáticos. En él se pusieron las bases fundamentales para el camino que han recorrido desde entonces las metodologías de primera persona y segunda persona.

Paralelamente a $O B A$, Varela y Jonathan Shear, filósofo y matemático, encabezaron otro proyecto más amplio. En una compilación de disímbolos trabajos de investigadores muy diversos, procedentes de distintos mundos y ambientes, pero con el común interés de explorar directa o indirectamente las vivencias, se logró ampliar la base para el desarrollo de metodologías de primera y segunda persona.

Depraz, Varela y Vermersch se unieron a un grupo de investigadores involucrados en lo que ya puede denominarse "metodologías de primera persona", agrupadas bajo tres grandes rubros: 1) el enfoque introspectivo derivado de la psicología científica, 2) el método de la reducción fenomenológica, derivada de la tradición filosófica de la Fenomenología (así con mayúscula) ${ }^{15}$ y de la psicología fenomenológica, y 3) la pragmática de la meditación, derivada de las tradiciones Budista y Védica. Los resultados de esta convocatoria se publicaron en The View from Within: First-Person Approaches to the Study of Consciousness (VFW) y quedaron resumidos en "First person Methodologies: What, Why, How?” (WWH), ambos con la autoría de Varela y Shear (1999).

En estas publicaciones se promovió el diseño de métodos rigurosos que permitieran a los investigadores recolectar descripciones de primera persona de la "experiencia vivida asociada con eventos cognitivos y mentales" (Varela \& Shear, 1999: p. 1), esto es, descripciones

15 Se pretendió diferenciar a la Fenomenología, con mayúscula, para destacarla como tradición filosófica. En ella la descripción 'fenomenológica' se basa necesariamente en la reducción, o actitud de suspensión y examen cuidadoso y metódico, con interrupción de la actitud natural. 
provistas por el sujeto que las vive. También se abordó la idea de que el "proceso que nos permite ser reflexivamente conscientes de nuestra experiencia y describirla tiene una estructura específica, y de que es posible e importante estudiarlo" (Petitmengin, 2009: p. 1).

Además de Varela, Depraz y Vermersch, se incluyeron trabajos de distintos autores sobre investigaciones y/o prácticas. Claire Peugeot abordó la experiencia intuitiva en relación con métodos introspectivos para la exploración de la experiencia. Carl Ginsburg escribió sobre la transformación somática, iniciada por Moshe Feldenkrais. De Andrew Bailey tenemos la colaboración sobre enfoques de William James acerca de la primera persona en los estudios del tiempo interno. Jean Naudin y colaboradores se refirieron al terreno de la comunicación humana en psiquiatría. Allan Wallace presentó ángulos pragmáticos de aspectos fundamentales de la tradición budista que mostraron la experiencia de siglos en el entrenamiento de la mente y el cultivo de las habilidades para la reflexión y la introspección. Aquí se destacaron serios problemas de interpretación y validación empírica. Jonathan Shear y Ronald Jevning abordaron los problemas mencionados en términos de las congruencias entre los informes verbales transculturales, y entre distintas tradiciones orientales, en relación con pretendidos exámenes de laboratorio de sus correlatos fisiológicos.

Esta variedad de enfoques permitió comprender la gama de tradiciones, prácticas e investigaciones involucradas. En $V F W$ merece resaltarse la estrategia semejante a la empleada en la creación de $O B A$, de conjuntar distintas visiones sobre los mismos problemas y propósitos. Pero en la creación de $V F W$ se analizan los enfoques de diversos grupos que trabajan sobre las metodologías de primera persona con acercamientos muy disímbolos, y se encuentran los elementos y rasgos comunes, las estructuras semejantes que se pueden extraer para tratar de unificar criterios y fundamentar metodologías sistemáticas.

Se encontró que todas las corrientes convocadas tenían la misma noción de método, o sea, un procedimiento claro para acceder al campo de la experiencia vivida, y también contaban con medios claros de expresión y validación "dentro de una comunidad de 
observadores que tuvieran familiaridad con el procedimiento" (Varela \& Shear, 1999: p. 6).

$\mathrm{Al}$ evaluarse los métodos de introspección, la Fenomenología y las meditaciones orientales, se concluyó que tenían estructuras nucleares comunes que se relacionaban con el contenido vivido de la experiencia y el proceso de examen por parte del sujeto. Se trataba entonces de distinguir el contenido y el proceso por el que aparece el contenido. Para esto se requería un momento de suspensión y redirección de la actitud natural, del contenido al proceso mental.

El sujeto (con o sin mediación) tiene una experiencia o contenido vivido (CV1), y se le pide que lo examine (describa, analice, y/o se haga consciente o aperciba, o preste atención). Este contenido se hará parte del contenido de una nueva experiencia, lo que será un nuevo contenido (CV2). Para que aparezca el par CV1-CV2, será necesaria "una redirección del pensamiento, una suspensión, una interrupción de la actitud natural, la cual normalmente no se detiene para acceder a sus propios contenidos" (Varela \& Shear, 1999: p. 8).

En esta estratificación, común a todos los métodos de primera persona, hay una distinción clara entre el contenido de un acto mental y el proceso a través del cual el contenido aparece. Los métodos analizados mostraron diferencias en este sentido. La introspección y la Fenomenología comprenden una atención enfocada al contenido, orientada a adquirir algún conocimiento o intelección (insight). Así el aprendizaje exitoso del método será concomitante a internalizar la duplicación de CV1 a CV2 con facilidad. Las tradiciones de meditación son más sutiles, y tienen menos preocupación por el contenido. Su meta es desarrollar el método hasta que se anule a sí mismo, produciendo estados de conciencia "pura" sin contenido (Varela \& Shear, 1999: p. 8).

El proceso que involucra el cambio de la actitud natural y busca directamente el nuevo contenido (CV2) moviliza una segunda fase que deja claro que a partir de la suspensión inicial el campo que estaba vacío "se vaya llenando progresivamente con nuevos datos fenomenales. Es el estado de descubrimiento, o de llenado experiencial, y esto requiere una disciplina sostenida para lograrse" (Varela \& Shear, 1999: p.8). Hay una aparente facilidad de acceso a la propia 
experiencia, pero el "llenado" con datos fenomenales que se hace posible por la suspensión tiene su propio tiempo de desarrollo y necesita un entrenamiento paciente.

En la comparación de tradiciones y métodos se encontraron dificultades que se trataron de superar a través de la mediación, consistente en la intervención de una segunda persona con el papel de "resonante empático". Con este hallazgo se pudo hablar ya de metodologías de segunda persona. Con variantes, se encontró también que en diversas corrientes se requirió una expresión y validación por medio de "informes susceptibles de retroalimentación intersubjetiva" (Varela \& Shear, 1999: p. 6).

La estrategia de Varela y sus colaboradores fue la de convocar diversas posturas y tradiciones dedicadas al examen de la experiencia subjetiva y encontrar los elementos comunes. Este mismo enfoque estratégico se continuó en la historia de estas metodologías y en su misma aplicación. ${ }^{16}$ Como ejemplo reciente, tenemos la búsqueda de "estructuras genéricas comunes" de datos fenomenológicos obtenidos en "entrevistas de explicitación" 17 en pacientes epilépticos. Las descripciones de señales de advertencia, obtenidas en las "entrevistas de explicitación”, pueden vincularse con datos neurofisiológicos. Estas aplicaciones se inician en los grupos fundados por Varela en Francia a fines de los años noventa, y persisten en la actualidad.

V.

Claire Petitmengin, integrante de los grupos de Neurofenomenología fundados en Francia, llevó a cabo otra publicación que marca un hito en la historia que estamos presentando. En el 2009, esta investigadora prologa y recopila, a diez años de la aparición de TheView from Within, los trabajos resultantes de las ramificaciones de los primeros esbozos de las metodologías que nos ocupan, y otras investigaciones

16 Algunos autores (Peirce, Eco) lo consideran un método abductivo.

17 Entrevistas donde se busca que el paciente "se haga consciente" de señales de advertencia que antecedan a las crisis epilépticas. 
relacionadas. En esa publicación se exponen los distintos caminos tomados, pero sorprende más el resultado convergente de rutas tan aparentemente diversas. Como ya se ha señalado, las metodologías de primera y segunda persona actualmente evolucionan en distintos campos, algunos vinculados con las neurociencias.

Ten Years of Viewing from Within: The Legacy of Francisco Varela (TY) (Petitmengin et al., 2009) apareció como conmemoración de la historia de las metodologías de primera persona. El objetivo fue examinar y refinar este programa de investigación sobre métodos de primera persona a través de contribuciones basadas en investigación empírica. Se trató de reunir investigadores que no sólo propusieron descripciones de primera persona, sino que trataron de "describir el proceso mismo de la descripción para hacer el proceso reproducible -una condición necesaria para cualquier comprensión científica” (Petitmengin, 2009: p. 7). Se aceptó que si los científicos mismos quieren estudiar sistemáticamente la experiencia vivida como se desenvuelve en la conciencia, necesitan práctica para volverse sus propios y mejores "instrumentos". El proceso de entrevistar a alguien sobre su experiencia vivida, un "enfoque de segunda persona" requiere un nivel significativo de familiaridad en el nivel de "primera persona” con la propia estructura de la experiencia del entrevistador que guía el proceso (Froese, 2011b: p. 255).

Al igual que en las obras precedentes, encontramos interrogantes sobre las formas de hacernos conscientes de nuestras experiencias, y de describirlas dentro de un método riguroso. Pueden detectarse así las estructuras genéricas resultantes de esas exploraciones y sus implicaciones epistemológicas.

Hay en $T Y$ avances y diferencias importantes respecto de las obras predecesoras. En ella se profundiza en el carácter señalado como prereflectivo (pre-reflective) e implícito de las vivencias, y desde el inicio del trabajo se hacen importantes señalamientos distintivos:

Por experiencia "pre-reflectiva" queremos decir una experiencia (por ejemplo de dolor) que se vive sin estar totalmente consciente de sí o auto-consciente. Usamos el término pre-reflectivo con el significado de que "no se reconoce a sí mismo". Sin embargo, 
la metáfora subyacente al espejo implica un distanciamiento que aquí no es relevante, y algunos autores en este número prefieren usar el término "pre-reflexivo" sólo para enfatizar la ausencia de auto-referencia. ${ }^{18}$ También es importante notar que según nuestra visión, la experiencia "reflectiva" no implica un estado mental particular que hiciera su objeto a la experiencia inicial (de dolor); ni tampoco involucra a un sí mismo (self), un ego, un ‘yo' o un sujeto, mientras que la experiencia pre-reflectiva sería sin yo y sin ego.

Sin embargo, en esta introducción y en todo el número publicado, la meta no es dar una definición definitiva y conceptual del término "pre-reflectivo" que sea completamente satisfactoria. $\mathrm{Ni}$ siquiera pretendemos que "pre-reflectivo" sea la palabra correcta para designar este aspecto de nuestra experiencia, no sólo por las connotaciones mencionadas antes, sino porque el "pre" parece implicar que la experiencia pre-reflectiva debe ser seguida siempre por la experiencia reflectiva. Nuestra falta de estar conscientes de lo que es más íntimo para nosotros - nuestra propia experiencia- es un gran misterio, que los estudios de la conciencia apenas empiezan a investigar (Petitmengin, 2009: p. 9).

Petitmengin enfatiza que la meta de la publicación conmemorativa fue señalar esa dimensión poco explorada e inducir a los lectores a detectarla. Asimismo se recalcó que éste era un campo de investigación de un inmenso potencial. Esta investigadora ha seguido trabajando en lo que llama "microgénesis de los pensamientos", que son las operaciones sutiles que llevamos a cabo para redireccionar la atención o traer una idea o pensamiento hacia la conciencia, y que también permiten construir la distinción entre el sí mismo y el otro. Esto sucede en forma "pre-reflectiva", con sólo una apercepción parcial de nuestro proceder (Anderson, 2007: p. 112). En diversos

18 Daniel Stern prefiere el término "pre-reflexivo", y su trabajo trata la cuestión del pasaje de la experiencia no verbal pre-reflexiva a la experiencia verbal reflexiva desde una perspectiva del desarrollo (Petitmengin, 2009: p. 16). 
trabajos, Petitmengin (2006, 2007a, 2007b, 2009b, 2011, 2013) ha seguido desarrollando estos temas.

En 2011, Petitmengin se refiere a técnicas para analizar la experiencia que en este caso denomina "no consciente", y señala que el uso de una palabra imbuida de "historia fenomenológica tal como 'pre-reflectivo' [...] da lugar al riesgo de mala interpretación o sobre-interpretación" (Petitmengin, 2011: p. 48). Por lo tanto decide usar el término "no reconocido (unrecognized)" al referirse a los rasgos de la experiencia de los cuales el sujeto no esté consciente.

En la publicación conmemorativa del 2009 también se revisa la idea de que en estas metodologías se proceda "desde adentro o desde el interior”. Se cuestiona la postura de que el auto-análisis de las vivencias sea realmente "desde adentro". Se ha encontrado que, extrañamente, mientras la atención está más separada de su concentración en los objetos, para entrar en contacto con la experiencia, se torna más reducida la distinción correspondiente entre el 'exterior' y el 'interior'. Lejos de presuponer esta distinción y considerarla como 'dada', en lugar de ello, la exploración de la experiencia "prereflectiva"(pre-reflective) nos lleva a interesarnos en la forma en que se constituye. Por lo tanto, los autores de estos trabajos no se consideran 'introspeccionistas', en el sentido de que tornamos nuestras miradas exclusivamente "hacia dentro" (Petitmengin, 2009: p. 12). ${ }^{19}$

La publicación de Petitmengin despertó interés en relación a diversos aspectos de las metodologías de primera y segunda persona. Sobre la validación de los datos fenomenológicos, se ha planteado que "las preguntas sobre el estudio de la experiencia vivida deben

19 Esta idea puede tener un parentesco con la tesis fenomenológica husserliana de la intencionalidad y la constitución. La intencionalidad, noción central, es una propiedad de las vivencias, y por ello es una propiedad subjetiva, "interna", que no puede verse ni descubrirse mirando el mundo externo sensible. Hay que mirar al interior para descubrirla. Se descubre que la vivencia es en su misma esencia referencia a algo, a otra cosa que no es conciencia, que no es vivencia. En la inspección fenomenológica se trata de ver en las vivencias intencionales, que sólo puedo descubrir en mi interior, que está formándose y constituyéndose continuamente de manera compleja y multiforme, el mundo externo, en el que vivimos diariamente. Esta es la tarea de la fenomenología constitutiva: describir la constitución, o sea la conformación del mundo en y para la vida de conciencia. (Comentario en comunicación personal de Antonio Zirión). 
tratarse de manera sistemática en relación con el dominio al que se relacionan, esto es, la experiencia vivida" (Froese, 2011: p. 255). Esto se relaciona con la irreductibilidad de los datos fenomenológicos a datos neurocientíficos. Froese, comentarista de Petitmengin, señala que no debe aceptarse el rechazo escéptico del uso de estos métodos considerándolo como "sesgado, no confiable y no científico, sobre la presunción a priori de que la introspección y la descripción de la experiencia vivida distorsionan las experiencias que se están informando" (Froese, 2011b: p.254). Puede ser el caso que una experiencia cambie en el proceso de hacerse consciente y describirla. ¿Cómo sabríamos que ese es el caso? ¿Podemos cuantificar o limitar la extensión hasta la cual se cambia la experiencia, y en realidad importa? Si es así, ¿en qué forma se minimiza cualquier interferencia dañina? (Froese, 2011b: p.254). Esto plantea rutas necesarias por donde proseguir las investigaciones.

Debe destacarse que ya Husserl, en Ideas I trata el tema de las dificultades de la "auto-observación" y los escepticismos metodológicos en la psicología empírica, haciéndolos extensivos a la fenomenología en cuanto su valor cognitivo. Planteó las relaciones internas y las congruencias entre la psicología y la fenomenología en el valor cognitivo, lo que no aminora el carácter de "ciencia de esencias" de la fenomenología. Es señalada la ventaja que tiene la fenomenología que "no tiene que hacer constataciones de existencia" sobre las vivencias, pero puede informar del "vivenciar el vivenciar". En esto se destaca el papel y valor cognitivo de la reflexión, que permite llegar a "conocimiento absolutamente válido" y sin alteración de las vivencias descritas. La conexión fenomenología/psicología plantea además una normatividad de la fenomenología hacia la psicología empírica (Husserl, 1913: pp. 259-268). ${ }^{20}$

Sobre la validación, en TY resalta la importancia de que los autores de las investigaciones puedan enfocar sus métodos hacia

20 Lo que Husserl viene a decir sobre este tema es que no puede admitirse una alteración meramente presunta de las vivencias debida a la reflexión u ocurrida a sus espaldas, sino que, para ser admisible, esta alteración tendría que ser ella misma vista, percibida; pero si lo es, entonces el argumento de la alteración cae por su propio peso, pierde toda validez. (Comunicación personal de Antonio Zirión). 
sí mismos, lo que resulta en una investigación de la conciencia de "segundo orden". Si se pretende que el método de estar consciente y describir la experiencia sea un método científico replicable, será necesario "hacerse consciente de" y describir el proceso mismo de hacerse consciente (Froese, 2011b: p. 254).

El número conmemorativo presentó a los investigadores que usan métodos prácticos concretos y disciplinados para hacerse conscientes de y describir la experiencia vivida, y al mismo tiempo describir su método. Sus contribuciones se organizaron alrededor de diferentes ejes de exploración de las vivencias.

El primer grupo de contribuciones se enfoca en la estructura del mero proceso de hacerse consciente de la propia experiencia vivida y su descripción. Incluye los dispositivos que permiten a los entrevistadores inducir el proceso y sobreponerse a sus dificultades. En el segundo grupo de trabajos se tienen ejemplos de "estructuras genéricas experienciales" identificadas en las descripciones de primera persona. Se destacan los procesos de intelección o insights para identificar esas estructuras. Todo se enfoca primordialmente sobre la estructura de la percepción (Petitmengin, 2009a: p. 15). El tercer grupo de participaciones aborda el tema de la transición de la conciencia pre-reflectiva a la reflectiva. Se enfoca desde las perspectivas de investigadores en procesos de desarrollo y psicoterapéuticos. Trata el uso de descripciones de la transición aludida para derivar sus consecuencias epistemológicas (Petitmengin, 2009a: p. 15).

Aunque hemos presentado el agrupamiento de temas, cabe aclarar que en cada rubro existe una gran diversidad de enfoques y estrategias para tratar el complejo tema de la experiencia subjetiva. En este texto conmemorativo se concluye que después de diez años de practicar la visión desde "el interior" ${ }^{21}$ el objetivo fue mostrar que las mismas "condiciones de posibilidad de la disciplina de "primera persona' ya se encuentran en el proceso de establecerse" (Petitmengin, 2009a: p.17).

21 Se aclara que se mantendrá la terminología de "visión interior" en homenaje a Varela, aunque nos parece objetable. 
En esa conclusión se considera que se están logrando métodos como procedimientos claros y que encaminan a formas de expresión y validación dentro de comunidades de observadores con práctica en procedimientos para acceder a ciertos dominios fenoménicos. También apuntan a logros en cuanto a métodos y lenguajes que le dan al investigador posibilidades de verificabilidad entre investigadores con niveles equiparables de entrenamiento y pericia (Petitmengin, 2009: p. 17).

En el 2009 ya se pudo reseñar la aplicación de las metodologías en desarrollo en el inicio de investigaciones en comunidades emergentes para diseñar y ejecutar proyectos de exploración de las diferentes dimensiones de la experiencia humana, ya sea identificando estructuras experienciales o articulando estructuras experienciales y neurofisiológicas (Petitmengin, 2009a: p. 17). Esto se refiere a los grupos franceses ya mencionados de investigación sobre epilepsia. Quedó explícita la importancia de las aplicaciones potenciales en diversos terrenos de la actividad humana como los pedagógicos y médicos.

Es importante actualizar esta historia de las metodologías tratadas. Se ha seguido trabajando en su refinamiento y posibilidades de validación (Petitmengin \& Bitbol, 2013), y más recientemente hubo logros importantes en cuanto a la consecución de confirmaciones experimentales (Petitmengin, 2013). Aunque en un principio Petitmengin no aceptaba la propuesta de Froese de confirmar sus propuestas con experimentos, recientemente lo hizo. Froese ha comentado que aunque todavía las metodologías muestran deficiencias, ya están encaminadas en la dirección correcta para promover su aceptación generalizada en protocolos científicos. ${ }^{22}$ Esto también nos parece un ejemplo importante de cooperación entre investigadores que muestran apertura y buena voluntad.

22 Comunicación personal de T. Froese. 
Comentario final

I. Hemos recorrido un trayecto de la historia de lo que, hoy en día, se consideran las metodologías de primera y segunda persona impulsadas por la Neurofenomenología. En virtud del inicio de la apertura a estas metodologías dentro de la neurociencia cognitiva hemos considerado que el conocimiento de la génesis y la evolución de estos medios de investigación esclarecerá sus aportes y ofrecerá la oportunidad de que nuevos investigadores se interesen en ellos.

Creemos que en la sucinta historia expuesta puede apreciarse la calidad epistémica que tienen y que continúan desarrollando las metodologías de primera y segunda persona de la Neurofenomenología, para su incorporación plena a protocolos neurocientíficos. Enfatizamos que pretendemos que ésta sea una respuesta a la evidente ignorancia, o franco rechazo, que todavía priva en la neurociencia cognitiva, principalmente anglo-sajona. Los méritos de la exploración sistemática iniciada en Occidente por Franz Brentano y Edmund Husserl, y seguida por un intenso movimiento fenomenológico en plena actividad, merecen ser conocidos y difundidos. Principalmente en algunos países anglo-sajones esto se ignora o se descalifica.

Nos interesa enfatizar el aprovechamiento que Varela inició de la tradición fenomenológica husserliana que conjuntó a los afanes de Vermersch para rehabilitar las técnicas introspectivas, trabajando en el fondo de las milenarias enseñanzas de las técnicas meditativas orientales.

El camino ha sido arduo, pues se trata de un terreno difícil que ofrece enormes retos y que está inexplorado en su mayor parte. Es el mundo de la subjetividad el que la ciencia moderna positivista vio inicialmente con temor y reservas sobre su confiabilidad, y que incluso negó por algún tiempo. No se cuenta aún con suficientes herramientas para su exploración, aunque en su historia encontramos poderosos elementos como el método fenomenológico husserliano, y las tradiciones milenarias orientales. Pero se requieren nuevas visiones que vislumbren el futuro sin ignorar el pasado. 
II. La figura de Francisco Varela, biólogo cognitivo y estudioso de la filosofía, ocupa un lugar importante en nuestras investigaciones. Hemos resaltado su afán por conjuntar conocimiento de diversas fuentes para seguir metas comunes. Partiendo de una "inspiración" en la fenomenología husserliana, pero con el fondo de interés teórico y práctico de las tradiciones meditativas orientales, Varela mostró que el trabajo conjunto en cooperación puede rendir frutos.

Queremos enfatizar que Varela tomó tradiciones filosóficas antiguas, pero las actualizó y las puso al servicio de los requerimientos de su tiempo. En lugar de exégesis, las tomó como punto de partida para construir en función de su época. Comunicó este espíritu a sus colaboradores para ver no sólo la historia de la filosofía, sino las posibilidades actuales para trabajar con los problemas de la ciencia cognitiva vigente.

Se arriesgó a lidiar con el método fenomenológico y a presentar una versión sobresimplificada, pero que pudo compararse con numerosas disciplinas y métodos afines. Trabajó junto a filósofos, antropólogos y psicoterapeutas. Dio muestras de las posibilidades enormes de la interdisciplinariedad en colaboraciones de apertura y renovados deseos de exploración.

III. Consideramos que hay una enseñanza digna de analizar en la historia de las metodologías emanadas de la Neurofenomenología, no sólo por el hallazgo epistemológico abductivo de lograr encontrar estructuras genéricas compartidas y un método común para "hacer conciencia de", sino por el valor que puede entrañar establecer vínculos fuera de la ciencia convencional. Nos muestra que no es aconsejable marginar posibles fuentes de conocimiento.

Desde el inicio en 1994 en que Varela, Vermersch y Depraz empezaron a colaborar fue ya notable la apertura del primero hacia la fenomenología, y deVermersch por recuperar la utilidad de técnicas introspectivas guiadas, parcialmente olvidadas y marginadas de la psicología convencional considerada científica. También ya hemos mencionado el fondo común de interés teóricos y prácticos de los tres investigadores en las tradiciones meditativas orientales. Pensamos que realmente fue este vínculo el que les permitió trabajar armónicamente. 
En la actualidad existe ya investigación neurocientífica en relación con diversos aspectos de las tradiciones meditativas orientales. El caso del biólogo molecular y monje Matthieu Ricard, quien se ha sometido a estudios de neuroimagen funcional durante diversos estados meditativos, es digno de mención. Estos hallazgos tienen gran difusión y plena aceptación en la comunidad científica. Sabemos que existen problemas interculturales para el pleno aprovechamiento de estos recursos, ya que esas técnicas, en general, no se ocupan de proporcionar informes de la experiencia subjetiva, sino que constituyen disciplinas espirituales. Creemos que es este mismo carácter el que las hace tener apertura y hacer propensos a sus seguidores a tener espíritu de cooperación y búsqueda de metas comunes.

Los casos del psicoanálisis y los distintos recursos psicoterapéuticos marginados en diversas áreas de las neurociencias merecen también analizarse a profundidad. Creemos que para que pudieran establecerse diálogos fructíferos y plantear arenas comunes de discusión se necesita que los bandos estén dispuestos realmente a escucharse mutuamente en actitudes abiertas y desinteresadas.

Resaltamos nuevamente la figura de Varela, quien privilegió los aspectos pragmáticos sobre los teóricos. Esto es evidente en los casos de algunos grupos de auto-ayuda de indudable éxito terapéutico y trascendencia social como el de los Alcóhólicos Anónimos y otros similares, donde, basicamente, se han aplicado estrategias para "hacer conciencia de", con resultados ya probados repetidamente.

No desconocemos la dificultad que implica para un científico abordar campos no legitimados por el llamado "método científico", pues sabemos lo difícil que ha sido erradicar el oscurantismo, la superstición y el pensamiento mágico como lo muestra la historia de la ciencia. Aún en el presente los medios de comunicación deforman y alteran situaciones de conocimiento, desvirtuándolas. Éste es un problema de enorme trascendencia social.

Creemos que en las ciencias sociales ha habido mayor apertura, y el caso de la antropología nos resulta paradigmático para comprender cómo pueden explorarse terrenos de la experiencia subjetiva sin abandondar posturas razonables en relación con la ciencia convencional. También pensamos que la filosofía debe contribuir a 
mantener un equilibrio y un sano espíritu de exploración epistemológica entre los innumerables campos del conocimiento. La importancia social de esto no debe soslayarse.

Las consideraciones previas no sólo son provechosas sino necesarias. En algunos campos de las ciencias cognitivas han aparecido conceptos como la "metacognición", que repiten con nueva terminología conceptos tan fundamentales de la fenomenología tradicional como la intencionalidad denominándola "aboutness". Con estos ejemplos se cumple una consideración futurista de Zahavi, quien, con la intención de promover los valores de la fenomenología, señaló que debía ser explorada por los actuales científicos cognitivos. Si no la conocían corrían el riesgo de creer descubrir algo que ya estaba descubierto desde mucho tiempo atrás (Zahavi, 2012).

Los grupos de investigación interdisciplinaria que cooperan de buena voluntad pueden ser el futuro para enriquecerse de fuentes de conocimiento no exploradas aún donde participen neurocientíficos de mentalidad abierta. ¿Será posible observar el trabajo conjunto y fructífero de un psicoterapeuta y un neurofisiólogo?

IV. El tema de la introspección es un ejemplo de una fuente de conocimiento que había sido considerada "no científica", por disparidades intersubjetivas en la historia de su génesis y evolución. Es un tema amplísimo y creemos que sigue siendo controversial, aunque su utilidad se sigue demostrando.

En el mismo artículo seminal de Varela de 1996, hay contradicciones sobre la participación de la introspección en la exploración de la experiencia subjetiva. Creemos que dentro de la misma fenomenología tradicional, que niega que sea introspección, quedan diversos aspectos que dilucidar. Sin embargo, en la génesis y evolución de las metodologías de primera y segunda persona se han tomado en cuenta todas esas dificultades. Vermersch ha sido el encargado de ir afinando el concepto al aplicarlo en las entrevistas de explicitación para recabar informes subjetivos que puedan tener validez como datos científicos.

En terrenos como la medicina psiquiátrica el tema todavía es debatido, pero se reconocen las bondades de algunos tipos de 
introspección. En cualquier caso, donde se emplee el término en la actualidad todavía resulta necesario hacer aclaraciones. Creemos que, en general, es un terreno pendiente de afinar pero que no merece descartarse y no debe emplearse a la ligera. Seguramente su plena aplicación en los terrenos de la ciencia cognitiva irá esclareciéndolo.

V. Idealmente consideramos que los avances en las metodologías de primera y segunda persona para elaborar material sobre la experiencia subjetiva, susceptible de incorporación a protocolos científicos, deben considerarse de forma transdisciplinar. Se trata de un problema que ha surgido a la luz de la ciencia cognitiva actual. Es un problema nuevo que debe afrontarse con estrategias nuevas y no convencionales. Idealmente, las ciencias cognitivas también parecen presentar la necesidad emergente de funcionar como un organismo coordinado nuevo sin obstáculos de barreras disciplinares, aunque creemos que aún no sucede así. Se podrá hablar ya no de ciencias cognitivas, sino de ciencia cognitiva, en singular.

Varela intentó fundar grupos de investigación que funcionaran en forma transdisciplinar, aunque nunca utilizó el término. Estos grupos son escasos y creemos que aún no han alcanzado el ideal de la transdisciplinariedad, pero eso debe ser una meta que no se pierda de vista. La realidad es que todavía se trabaja en forma interdisciplinar con numerosas deficiencias.

Por la naturaleza epistémica del problema de la interdisciplinariedad, al analizar el desarrollo histórico-epistémico de las metodologías de primera y segunda persona impulsadas dentro de la Neurofenomenología, se detectan numerosos problemas dentro de confrontaciones interdisciplinares como la mezcla de terminologías y discursos, diversas visiones sobre un mismo objeto de estudio, y posiciones aparentemente irreductibles.

Varios de estos problemas tienen aspectos positivos y negativos. Creemos que si sobre un mismo objeto de estudio hay debates y discrepancias, se enriquecen las posibilidades de un mejor análisis. Ello requiere, por supuesto, diálogos y debates entre posiciones, con un necesario espíritu de cooperación y conciliación. Este espíritu sí ha 
prevalecido en los grupos convocados por la Neurofenomenología. Ella misma, que va logrando avances, es un ejemplo de cooperación interdisciplinar, pues la idea original de Varela fue la de comunidades de investigación en las que el conocimiento y la investigación se compartieran. En un análisis cuidadoso de ese programa de investigación podemos ver que sus fundamentos presentan aparentes oposiciones entre sus bases teóricas, pero que sus enfoques pragmáticos parecen superar esas oposiciones.

Creemos que en todo esto nuevamente destaca el papel que ha jugado un espíritu filosófico, y pensamos que lo que algunos podrían considerar terrenos borrosos entre la ciencia y la filosofía, pueden resultar zonas de frontera o limítrofes donde puede florecer el conocimiento. Los problemas de las ciencias cognitivas son un ejemplo del trabajo en estas zonas con conceptos como "conciencia", que fueron casi privativos de la filosofía y ahora se tienen que investigar en forma compartida.

VI. Consideramos que es adecuado concluir con una postulación de aspectos que pueden dar lugar a diferentes líneas de investigación.

En primer lugar queremos destacar la importancia de aquilatar la historia como una fuente epistémica. En la confrontación de disciplinas, algunos sectores descalifican otros campos sin valorar sus posibilidades. La historia que hemos presentado y analizado nos ha mostrado la utilidad de la diversidad de fuentes de conocimiento. Por lo tanto, consideramos relevante profundizar en los escollos que representan prejuicios y sesgos para el aprovechamiento de fuentes de conocimiento consideradas como "marginales", "no legitimadas", "no científicas", etc. El caso de la ya una vez desechada "introspección”, muestra la importancia de contextualizar los hallazgos de épocas pasadas, y no descartar sus potencialidades.

Consideramos que es necesaria una investigación constante para revalorar las condiciones en que puede ser productiva la interdisciplinariedad, y destacar las situaciones en que se requiere una verdadera transdisciplinariedad ante problemas inéditos y sin antecedentes. El campo es vasto, pero las oportunidades de cosechar conocimiento son inagotables. 


\section{REFERENCIAS BIBLIOGRÁFICAS:}

Anderson, Walter Truett. 2007. "Bringing Experience out of the Closet. Reflections on the Conference on First-Person Methodologies in the Study of Consciousness" en Journal of Consciousness Studies, vol. 14, no. 8, pp. 110-16.

Capuccio, M. (ed.). 2006. Neurofenomenologia: le scienze della mente e la sfida dell'experienza cosciente. Milano: Bruno Mondadori.

Dennetr, Daniel. 2001. "The Fantasy of First Person Science”, version electrónica, consultada en: http: / / ase.tufts.edu/cogstud/papers/chalamersdeb3dft.htm

Depraz, Nathalie (et al.)(eds.) 2003. On Becoming Aware. A pragmatics of experiencing. Amsterdam: John Benjamins Publishing Company.

DíAz, José Luis. 2007. La conciencia viviente. México: Fondo de Cultura Económica.

Díaz, José Luis. 2013. “A Narrative Method for Consciousness Research” en Frontiers in Human Neuroscience, v. 7 (n. 739), pp.1-12.

FriTh, Christopher D. 2012. "Disorders of Conscious and Unconscious Mental Processes” en Eric Kandel. Principles of Neural Science. New York: McGraw-Hill, pp.1383-1384.

Froese, T. 2013. "Interactively guided introspection is getting science closer to an effective consciousness meter" en Consciousness and Cognition ,22, 672-676.

Froese, T. (et al.) 2011a. "Validating and Calibrating First- and Second-Person Methods in the Science of Consciousness" en Journal of Consciousness Studies, v. 18, n. 2, pp.38-64.

Froese, T. (et al.) 2011b, "Reviewing from Within. A Commentary on First- and Second-Person Methods in the Science of Consciousness" en Constructivist Foundation, v. 6, n.2, pp. 254-269.

Gallagher, S. \& Dan Zahavi. 2013. La mente fenomenológica. Una introducción a la filosofía de la mente y a la ciencia cognitiva. Madrid: Alianza Editorial. Traducción de Marta Jorba Grau.

GladZiejewski, Pawel. 2010. "Neurophenomenology: an invitation to discussion” en AVANT, www.avant.umk.pl

Hurlburt, R.T. \& Heavey C.L. 2006. “The Descriptive Experience Sampling method” en Phenomenology and the Cognitive Sciences, v. 5, nn. 3-4, pp. 271-301.

HuSSERL, E. 1913. Ideas relativas a una fenomenología pura y una filosofía fenomenológica. Libro Primero.Introducción general a la fenomenología pura. México, Fondo de Cultura Económica / Universidad Nacional Autónoma de México, 2013. Nueva edición y refundición integral de la traducción de José Gaos por Antonio Zirión Quijano. 
Kandel, Eric (et al.) (eds.) 2012. Principles of Neural Science. New York: McGraw-Hill.

Laughuin, Charles D. (et al.) 1990. Brain, Symbol \& Experience. New York: Columbia University.

Le Van Quyen, Michel. 2010. "Neurodynamics and Phenomenology in Mutual Enlightment: The Example of the Epileptic Aura” en Stewart, J. (et al.) 2010. Enaction. Toward a New Paradigm for Cognitive Science. Cambridge: The MIT Press, pp. 245-265.

Lutz, A. 2002. "Toward a Neurophenomenology as an Account of Generative Passages: A First Empirical Case Study" en Phenomenology and the Cognitive Sciences, v. 1, pp. 133-167.

Maturana, H.R. y Francisco Varela. 1998, The Tree of Knowledge. Boston: Shambhala.

Petitmengin C. 1999. "The intuitive experience” en The View from Within: First-person Approaches to the Study of Consciousness, a special issue of the Journal of Consciousness Studies, v.6, nn. 2-3, pp. 43-77.

Petitmengin C. 2006, "Describing One's Subjective Experience in the Second Person: An Interview Method for the Science of Consciousness" en Phenom. Cogn. Sci., v. 5, pp. 229-269.

Petitmengin C. (et al.) 2007A. “Anticipating Seizure: Pre-reflective Experience at the Center of Neuro-phenomenology” en Consciousness and cognition, v.16, n. 3, pp. 746-764.

Petitmengin C. 2007в. "Towards the Source of Thoughts. The Gestural and Transmodal Dimension of Lived Experience" en Journal of Consciousness Studies, v. 14, n. 3, pp. 54-82.

Petitmengin, C. 2009a. "Editorial Introduction", en Ten Years of Viewing from Within: The Legacy of Francisco Varela. Journal of Consciousness Studies, v. 16, nn.10-12, pp. 7-19.

Petitmengin C. (ET al.) 2009b. "Listening from within", Journal of Consciousness Studies, vol.16, no. 10-12, pp. 252-284.

Petitmengin, C. 2011. "Describing the Experience of Describing? The Blindspot of Introspection” en Journal of Consciousness Studies, v. 18, n. 1, pp. 44-62.

Petitmengin, C. (et al.) 2013. "A gap in Nisbett and Wilson’s findings? A first-person access to our cognitive proceses" en Consciousness and Cognition, 22, 654-659.

Petrtmengin, C. y Michel Bitbol. 2013. "On the Possibility and Reality of Introspection” en Kairos, v. 6, pp. 173-198.

Rudrauf, David (et al.) 2003. "From Autopoiesis to Neurophenomenology: Francisco Varela's Exploration of the Biophysics of Being”, Biol Res vol.36,pp.27-65.

Thompson, E. 2004. "Life and Mind: From Autopoiesis to Neurophenomenology. A Tribute to Francisco Varela" en Phenomenology and the Cognitive Sciences, vol. 3, pp. 381-398. 
VArela, Francisco. 1988. Conocer. Las ciencias cognitivas: tendencias y perspectivas. Cartografía de las ideas actuales. Barceloa: Editorial Gedisa. Traducción: Carlos Gardini..

VARela, Francisco. 1996. "Neurophenomenology: A Methodological Remedy for the Hard Problem” en Journal of Consciousness Studies, v. 3, n. 4, pp. 330-349.

Varela, Francisco y Jonathan Shear (eds.). 1999. "First Person Methodologies: What, why, how?" en TheView fromWithin: First Person Approaches to the Study of Consciousness, a special issue of the Journal of Consciousness Studies, vol. 6, nn. 2-3, pp.1-14.

Varela, Francisco Javier, Evan Thompson y Eleanor Rosch. 1991, De cuerpo presente. Las ciencias cognitivas y la experiencia humana. Barcelona: Editorial Gedisa, 2005. Traducción de Carlos Gardini. 